\title{
Beyond the Binary: Differences in Eating Disorder Prevalence by Gender Identity in a Transgender Sample
}

\author{
Elizabeth W. Diemer, ${ }^{1}$ Jaclyn M. White Hughto, ${ }^{2,3}$ Allegra R. Gordon, ${ }^{4,5}$ Carly Guss, ${ }^{4,6}$ \\ S. Bryn Austin, $4,5,7$ and Sari L. Reisner ${ }^{1,2,6,7, *}$
}

\begin{abstract}
Purpose: To investigate whether the prevalence of eating disorders (EDs) differs across diverse gender identity groups in a transgender sample.

Methods: Secondary analysis of data from Project VOICE, a cross-sectional study of stress and health among 452 transgender adults (ages 18-75 years) residing in Massachusetts. Age-adjusted logistic regression models were fit to compare the prevalence of self-reported lifetime EDs in female-to-male (FTM), male-to-female (MTF), and gender-nonconforming participants assigned male at birth (MBGNC) to gender-nonconforming participants assigned female at birth (FBGNC; referent).

Results: The age-adjusted odds of self-reported ED in MTF participants were 0.14 times the odds of self-reported ED in FBGNC participants ( $p=0.022$ ). In FTM participants, the age-adjusted odds of self-reported ED were 0.46 times the odds of self-reported ED in FBGNC participants, a marginally significant finding ( $p=0.068$ ). No statistically significant differences in ED prevalence were found for MBGNC individuals.

Conclusions: Gender nonconforming individuals assigned a female sex at birth appear to have heightened lifetime risk of EDs relative to MTF participants. Further research into specific biologic and psychosocial ED risk factors and gender-responsive intervention strategies are urgently needed. Training clinical providers and ensuring competency of treatment services beyond the gender binary will be vital to addressing this disparity.
\end{abstract}

Keywords: eating disorders; mental disorders; gender; transgender

\section{Introduction}

Research suggests that transgender people, whose assigned sex at birth does not match their gender identity, are more likely than cisgender people, whose assigned sex at birth matches their gender identity, to have been diagnosed with an eating disorder (ED) or to engage in disordered eating. ${ }^{1-4}$ Studies of college students and adolescents have found that odds of past year ED and past month ED symptoms were significantly higher than the odds of these behaviors in cisgender males, although comparisons to cisgender females have yielded inconsistent results. ${ }^{1,4}$ In addition, cisgender women, but not men, with reported gender identity conflict, report more disordered eating behaviors than cisgender women without gender identity conflict. ${ }^{2}$ Small studies have also found that transgender individuals report more disordered eating behaviors than healthy controls, but less than individuals with diagnosed EDs. ${ }^{5-7}$

\footnotetext{
${ }^{1}$ Department of Epidemiology, Harvard T.H. Chan School of Public Health, Boston, Massachusetts.

${ }^{2}$ The Fenway Institute, Fenway Health, Boston, Massachusetts.

${ }^{3}$ Yale School of Public Health, New Haven, Connecticut.

${ }^{4}$ Division of Adolescent and Young Adult Medicine, Boston Children's Hospital, Boston, Massachusetts.

${ }^{5}$ Department of Social and Behavioral Sciences, Harvard T.H. Chan School of Public Health, Boston, Massachusetts.

${ }^{6}$ Division of General Pediatrics, Boston Children's Hospital, Boston, Massachusetts.

${ }^{7}$ Department of Pediatrics, Harvard Medical School, Boston, Massachusetts.
} 
The term transgender encompasses a range of gender identities, including binary identities like female-to-male (FTM) or transgender men and male-to-female (MTF) or transgender women, as well as nonbinary identities such as gender nonconforming, genderqueer, and gender variant. ${ }^{8}$ Despite this heterogeneity, most literature on EDs in transgender populations fail to differentiate between transgender individuals with different gender identities. Only one previous study on EDs has been able to differentiate between participants with binary and nonbinary gender identities. ${ }^{3}$ That study, which collapsed all nonbinary individuals into a single category, found that, in transgender young adults, the unadjusted prevalence of past year self-induced vomiting was higher in nonbinary individuals relative to individuals with a binary FTM gender identity, and past year fasting was higher in individuals with a binary MTF gender identity relative to nonbinary individuals. ${ }^{3}$ However, nonbinary individuals who fall along a FTM gender identity spectrum may differ dramatically in exposures and behaviors from nonbinary individuals who fall along a MTF spectrum identity. Collapsing gender identity subgroups into larger categories could mask potentially significant differences in ED prevalence across transgender samples, which may have implications for intervention design, development, and implementation. To this point, no study has examined the prevalence of disordered eating or ED diagnosis across both gender identity spectrum and binary versus nonbinary gender identity. The aim of this study was therefore to investigate whether risk of self-reported ED and ED diagnosis in transgender adults differs across specific gender identity subgroups.

\section{Methods}

Participants and procedures

Participant data were derived from Project VOICE (Voicing Our Individual and Community Experiences), a community-based sample of 452 transgender and gender nonconforming Massachusetts residents ages 18 to 75 years, collected between August 2013 and December 2013. The majority of respondents $(88 \%)$ were sampled online through transgender electronic listservs, e-mails, web postings on communitybased websites, and social networking sites, and $12 \%$ were sampled in-person (through electronic tablets provided by the research team at transgender community events, local social programming, and other gatherings). Individuals were considered eligible for study participation if they were 18 years of age or older, self-identified as transgender or gender nonconforming, lived in Massachusetts for at least 3 months in the last year, and had the ability to read and write in either English or Spanish.

Using a participatory population perspective to work "with" not "on" transgender communities, the Project VOICE survey instrument and data collection plan were created by a team of community-based advocates, transgender leaders, researchers, and sexual and gender minority policy experts. ${ }^{9}$ The survey was designed for a fifth grade reading level. To ensure the integrity and validity of data collected, the survey followed best practices for Internet research with transgender people, including initial usability, pilot testing, and quality management processes to ensure unduplicated responses and valid study respondents. ${ }^{10}$ More detailed descriptions of the instrument construction and data collection process are available elsewhere. ${ }^{11}$ Project VOICE was approved by the Fenway Health Institutional Review Board.

\section{Measures}

ED diagnosis. Participants were asked to check all boxes that applied to the health conditions "anorexia nervosa or bulimia nervosa," queried as a single question. Four response options were provided: (1) a doctor, nurse, or other health professional has diagnosed me with this condition, (2) I think I have this health condition, (3) I do not have this health condition, and (4) I don't know if I have this health condition. Other Diagnostic and Statistical Manual of Mental Disorders, fifth edition (DSM-5) feeding or EDs were not assessed. For these analyses, two outcome variables were constructed. First, respondents were coded as having self-reported anorexia nervosa (AN) or bulimia nervosa (BN) if they marked the boxes "I think I have this health condition" or "A doctor, nurse, or other health professional has diagnosed me with this condition." Second, participants were coded as having an ED diagnosis if they marked "A doctor, nurse, or other health professional has diagnosed me with this condition." Although this lifetime ED screening is brief, previous research has found that, in population samples, single item measures of self-reported $\mathrm{AN}$ and $\mathrm{BN}$ have similar sensitivity and specificity to established, longer form screening tools. ${ }^{12}$

Gender identity. Gender was assessed using a twostep method ${ }^{13}$ asking (1) assigned sex at birth (female, 
male) and (2) current gender identity (man, woman, FTM/trans man, MTF/trans woman, genderqueer, gender variant, gender nonconforming, other gender). The two items were cross-tabulated to categorize participants according to current gender identity and assigned sex at birth. Specifically, a gender variable with four categories was constructed: MTF, FTM, gendernonconforming participants assigned male at birth (MBGNC), and gender-nonconforming participants assigned female at birth (FBGNC). Participants were also categorized as having a binary gender identity (male/trans man/FTM, female/trans woman/MTF) or a nonbinary gender identity (genderqueer, gender variant, gender nonconforming) based on their response to the current gender identity item and sex assigned at birth.

Age

Due to a limited number of outcomes in our sample, we were only able to control for age in our analyses. Age was measured continuously in years.

\section{Demographics}

Demographic characteristics are provided to better describe the nature of the study sample. Race and ethnicity were measured as separate items using a measure from the 2012 Behavioral Risk Factor Surveillance System Questionnaire and recoded into the following categories: White non-Hispanic, black non-Hispanic, Hispanic/Latino, other race/ethnicity non-Hispanic, and multiracial non-Hispanic. ${ }^{14}$ Educational attainment was measured on a five-point scale (1-5) with the following response options: less than high school education, high school diploma, some college, 4-year college degree, and graduate school. Employment status was queried with the question "Are you currently.??" with response options employed for wages, selfemployed, unemployed $1+$ years(s), unemployed $<1$ year, homemaker, student, and retired. For descriptive purposes, this variable was coded into employed (employed for wages or self-employed) and not employed (unemployed $1+$ years(s), unemployed $<1$ year, homemaker, student, retired). Access to gender affirmative medical services was queried with the question "Have you accessed any transgender-related medical interventions to affirm your gender (e.g., hormones, surgeries)?" (yes, no).

\section{Data analysis}

Statistical analyses were performed in SAS 9.4. Missingness in this sample was differential, ${ }^{11}$ violating the missing completely at random assumption required for valid statistical inferences using complete case analysis. ${ }^{15}$ Because of this violation, all survey variables with missing data were multiple imputed. Approximately $7.25 \%$ of participants were missing data on ED diagnosis, and $0.33 \%$ were missing data on gender identity. A fully conditional specification ${ }^{16,17}$ imputation method was used, consistent with previous research in transgender health. ${ }^{18}$ All subsequent analyses were conducted in the imputed dataset. Univariate statistics was obtained to characterize the frequency and distribution of ED diagnosis in the sample and by gender identity subgroups.

To assess the odds of ED diagnosis across gender identity categories, we fit a multivariable Firth logistic regression model, regressing $\mathrm{ED}$ diagnosis (yes, no) on gender identity (MTF, FTM, MBGNC, FBGNC, referent: FBGNC) and linear age. FBGNC contained the most participants and was therefore chosen as the referent group. Additional Firth logistic regressions, restricting the outcome to participants who reported having been diagnosed with an ED by a health professional, were also conducted as a sensitivity analysis. Because no individuals in the MBGNC strata reported ED diagnosis by a health professional, two separate regressions were run, one regressing $\mathrm{ED}$ diagnosis on age and gender nonconformity (nonbinary vs. binary gender identity) and one regressing ED diagnosis on age and gender spectrum (trans masculine vs. trans feminine). The Firth approach, which reduces distortion due to small effective sample size, was chosen for this analysis because of the rarity of ED diagnosis in our sample, particularly among MTF participants. ${ }^{19}$

\section{Results}

Demographic characteristics of the sample

Demographic characteristics of respondents are presented in Table 1 . Participants $(n=452)$ ranged in age from 18 to 75 years, with $34.1 \%$ between ages 18 and $24,21.3 \%$ between ages 25 and 30 , and $44.9 \%$ age $30-75$. The majority of participants (79.3\%) were non-Hispanic white, and $85.7 \%$ had a high school education or higher. Most participants (63.1\%) were currently employed. More than half $(55.0 \%)$ of the sample previously accessed gender-affirmative medical interventions (hormones and/or surgery).

Almost one third (31.5\%) of the sample was assigned a female sex at birth and identified as gender nonconforming or another nonbinary gender identity, and $31.3 \%$ were assigned a female sex at birth and identified 
Table 1. Demographic Characteristics of Project VOICE Participants $(n=452)$

\begin{tabular}{|c|c|c|c|c|c|}
\hline & $\begin{array}{c}\text { Total } \\
(n=452) n(\%)\end{array}$ & $\begin{array}{c}\text { FBGNC } \\
(n=143) n(\%)\end{array}$ & $\begin{array}{c}\text { FTM } \\
(n=142) n(\%)\end{array}$ & $\begin{array}{c}\text { MTF } \\
(n=127) n(\%)\end{array}$ & $\begin{array}{c}\text { MBGNC } \\
(n=42) n(\%)\end{array}$ \\
\hline Diagnosis of anorexia nervosa or bulimia nervosa & $21(4.7)$ & $14(9.7)$ & $6(4.1)$ & $1(2.8)$ & $0(0.0)$ \\
\hline $\begin{array}{l}\text { Self-reported anorexia nervosa or bulimia nervosa } \\
\text { Mean age (years) }\end{array}$ & $31(7.4)$ & $20(15.0)$ & $8(6.1)$ & $1(0.8)$ & $2(5.5)$ \\
\hline \multicolumn{6}{|l|}{ Race/ethnicity } \\
\hline White non-Hispanic & $361(79.3)$ & $117(81.8)$ & $114(80.2)$ & 95 (74.9) & $34(81.0)$ \\
\hline Black & $13(2.9)$ & $0(0.0)$ & $5(3.5)$ & $6(4.8)$ & $2(4.7)$ \\
\hline Hispanic & $43(9.5)$ & $11(7.7)$ & $11(7.7)$ & $16(12.6)$ & $5(11.9)$ \\
\hline Other & $14(3.1)$ & $4(2.9)$ & $3(2.3)$ & $7(5.4)$ & $0(0.0)$ \\
\hline Multiracial & $24(5.3)$ & $11(7.7)$ & $9(6.3)$ & $3(2.4)$ & $1(2.4)$ \\
\hline \multicolumn{6}{|l|}{ Education } \\
\hline$<$ High school & $13(2.9)$ & $2(1.4)$ & $4(2.8)$ & $5(4.0)$ & $2(4.8)$ \\
\hline High school diploma & $52(11.5)$ & $8(5.6)$ & $8.45(12.0)$ & $27(21.6)$ & $5(11.9)$ \\
\hline Some college & $134(29.5)$ & $47(32.9)$ & $37(26.1)$ & 39 (31.2) & $11(26.2)$ \\
\hline Four year college & $154(33.9)$ & $50(35.0)$ & $57(40.1)$ & $32(25.6)$ & $13(31.0)$ \\
\hline Graduate school & $101(22.3)$ & $36(25.2)$ & $32(22.5)$ & $22(17.6)$ & $11(26.2)$ \\
\hline Had accessed gender affirmative medical services & $250(55.0)$ & $38(31.8)$ & $111(84.1)$ & $89(70.4)$ & $11(42.6)$ \\
\hline Employed & $287(63.1)$ & $100(69.8)$ & $101(71.0)$ & $58(45.6)$ & $28(66.5)$ \\
\hline
\end{tabular}

FBGNC, gender-nonconforming participants assigned female at birth; FTM, female-to-male; MBGNC, gender-nonconforming participants assigned male at birth; MTF, male-to-female.

as a binary identity (e.g., man, male, or FTM). Just less than $10 \%$ of participants $(9.3 \%)$ were assigned a male sex at birth and identified as gender nonconforming or another nonbinary gender identity, and 27.9\% were assigned a male sex at birth and identified as having a binary gender identity (woman, female, or MTF).

\section{ED diagnosis}

Overall, $7.4 \%(n=31)$ of participants responded that they thought they had an ED or that a health professional had diagnosed them with an ED in their lifetime. The prevalence of ED diagnosis by a health professional in the sample was $4.7 \%(n=21)$. The results of an age-adjusted multivariable logistic regression on self-reported ED across categories of gender identity are presented in Table 2. The odds of reporting an ED among MTF participants were 0.14 times the odds of reporting an ED in FBGNC participants.

Table 2. Association of Gender Identity and Age with Self-Reported Anorexia Nervosa or Bulimia Nervosa in Project VOICE Participants $(n=452)$

\begin{tabular}{lccc}
\hline & $\begin{array}{c}\text { Firth penalized } \\
\text { odds ratio }\end{array}$ & $\begin{array}{c}\text { 95\% confidence } \\
\text { interval }\end{array}$ & $\boldsymbol{p}$ \\
\hline $\begin{array}{l}\text { Gender identity } \\
\text { FBGNC }\end{array}$ & 1.00 (referent) & & \\
FTM & 0.46 & $(0.20-1.01)$ & 0.068 \\
MTF & $\mathbf{0 . 1 4}$ & $(\mathbf{0 . 0 3 - 0 . 7 6 )}$ & $\mathbf{0 . 0 2 2}$ \\
MBGNC & 0.59 & $(0.13-2.36)$ & 0.36 \\
Age & 0.96 & $(0.92-0.98)$ & 0.11 \\
\hline
\end{tabular}

Firth penalized odds ratio $=$ Firth logistic regression uses penalized maximum likelihood to mitigate bias resulting from rare events. Odds ratios significant at $p<0.05$ are shown in bold.
MBGNC and FTM individuals also had lower odds of endorsing having had an ED than FBGNC participants, although these relationships were not statistically significant.

Table 3 presents the results of an additional model comparing the prevalence of ED diagnosis by a health professional across nonbinary versus binary gender identities, and a second model comparing ED diagnosis

Table 3. Associations of Gender Identity and Age with Diagnosis of Anorexia Nervosa or Bulimia Nervosa in Project VOICE Participants $(n=452)$

\begin{tabular}{|c|c|c|c|}
\hline & $\begin{array}{c}\text { Firth } \\
\text { penalized } \\
\text { odds ratio }\end{array}$ & $\begin{array}{c}95 \% \\
\text { confidence } \\
\text { interval }\end{array}$ & $p$ \\
\hline \multicolumn{4}{|l|}{ Model 1} \\
\hline \multicolumn{4}{|l|}{$\begin{array}{l}\text { Nonbinary gender } \\
\text { identity and age }\end{array}$} \\
\hline $\begin{array}{l}\text { Nonbinary gender identity } \\
\text { vs. binary gender identity }\end{array}$ & 3.16 & $(1.18-8.47)$ & 0.022 \\
\hline Age & 0.96 & $(0.91-1.02)$ & 0.19 \\
\hline \multicolumn{4}{|l|}{ Model 2} \\
\hline \multicolumn{4}{|l|}{ Gender spectrum and aqe } \\
\hline $\begin{array}{l}\text { Trans masculine vs. } \\
\text { trans feminine }\end{array}$ & 4.03 & $(0.74-21.86)$ & 0.11 \\
\hline Age & 0.97 & $(0.92-1.03)$ & 0.29 \\
\hline
\end{tabular}

Nonbinary gender identity $=$ Nonbinary gender identities (e.g., genderqueer, gender variant, gender nonconforming) compared to binary gender identities (man, woman, FTM, MTF).

Gender spectrum =One's location within a range of gender identities and sexes assigned at birth. Trans masculine (assigned female at birth and identify as: FTM, transgender man, trans male, gender nonconforming, or gender variant) compared to trans feminine (assigned male sex at birth and identify as: MTF, transgender woman, trans female, gender nonconforming, or gender variant).

Firth penalized odds ratio $=$ Firth logistic regression uses penalized maximum likelihood to mitigate bias resulting from rare events. Odds ratios significant at the $p<0.05$ level are shown in bold. 
in trans masculine (assigned female sex at birth) and trans feminine (assigned male sex at birth) respondents. For individuals with nonbinary gender identities, the odds of being diagnosed with an ED by a health professional were 3.16 times the odds of being diagnosed with an ED in individuals with a binary gender identity. The odds of ED diagnosis were also higher among individuals assigned a female sex at birth relative to those assigned a male sex at birth, although these results did not achieve significance at the $p<0.05$ level; this suggests that, consistent with our original model, the prevalence of ED diagnosis by a health professional may be higher in FBGNC individuals than in other gender identity subgroups. In addition, the results of this analysis suggest that differences in the prevalence of EDs in this sample may be driven primarily by differences between individuals with binary versus nonbinary identities, rather than between trans masculine and trans feminine participants.

\section{Discussion}

ED prevalence was unevenly distributed across gender identity groups in this community sample of transgender adults. The odds of self-reported ED in MTF participants were 0.14 times the odds in FBGNC participants, and the odds of self-reported ED in FTM participants were 0.46 times the odds in FBGNC participants. These results are somewhat inconsistent with a previous study on disordered eating behaviors among transgender individuals, which found increased prevalence of attempts to lose weight by fasting in MTF participants relative to nonbinary individuals, although that study did report increased prevalence of past-year self-induced vomiting in nonbinary individuals relative to FTM individuals, similar to our results comparing FTM and FBGNC. ${ }^{3}$ It is possible that our contrasting findings are a result of differences in the prevalence of disordered eating behaviors relative to full clinical disorders, or of variation in ED prevalence, knowledge, or access to care across populations.

Our finding of increased odds of self-reported ED in FBGNC participants may be explained through several mechanisms. A growing body of literature suggests that transgender people face stigma related to their gender identity at the individual, interpersonal, and structural levels, resulting in worsened physical and mental health. ${ }^{20}$ Indeed, a recent study in transgender young adults found that high levels of enacted stigma were associated with past year disordered eating, even after adjusting for social support. ${ }^{3}$ One pathway through which stigma impacts health among transgender people is avoidant coping (i.e., the employment of strategies, including disordered eating, that allow individuals to avoid stressors), as research documents an association between victimization and avoidant coping in transgender adults. ${ }^{21}$ In addition, research finds that transgender people with lower visual conformity (i.e., who are more visually gender nonconforming) are more vulnerable to mistreatment and worse health than transgender people with high visual conformity. ${ }^{11,22}$ Given prior research, it is possible that nonbinary participants in the sample may be less visually gender conforming (other people can tell they are transgender) than binary transgender people and, thus, experience more discrimination, which in turn could increase their risk of ED.

Gender socialization may also contribute to the development of EDs in transgender populations. ${ }^{23}$ The association between gender identity and reported EDs could be due to differences in the use of disordered eating to suppress gendered features or to conform to gendered body norms. Qualitative studies have suggested that FTM individuals may use restriction and bulimic behaviors to suppress breasts and hips and that MTF individuals engage in disordered eating to accentuate their femininity and to increase ability to be perceived as cisgender women, thus reducing risk of being targeted for violence and discrimination. ${ }^{9,24-26}$ Yet, in our sample, FBGNC participants were more likely than MTF participants to report an ED and marginally more likely than FTM participants to report an ED. This may indicate that FBGNC participants are striving to alter gendered features to a greater extent than other transgender groups or that the relationship between body dissatisfaction and EDs is moderated by some factor associated with gender identity in transgender adults.

When responses were restricted to participants who reported being diagnosed with an ED by a health professional, nonbinary participants were significantly more likely than participants with a binary gender identity to report an ED diagnosis. These results are generally consistent with our first model, although differences in ED prevalence by gender identity spectrum were not found to be significant at the $p<0.05$ level. This finding could suggest that differences in ED prevalence are driven primarily by differences between individuals with binary and nonbinary identities, rather than differences in gender identity spectrum. Future exploration of diagnosed ED by gender identity should 
be undertaken using a large sample to determine if a similar and statistically significant relationship is observed.

Finally, the prevalence of self-reported ED in this sample was 7.4\%. Prior studies of cisgender Americans have found a lifetime AN prevalence of $0.3 \%$ in males and $0.9 \%$ in females and lifetime $\mathrm{BN}$ prevalence of $0.5 \%$ in males and $1.5 \%$ in females using diagnoses based on Diagnostic and Statistical Manual of Mental Disorders, fourth edition (DSM-IV). ${ }^{27}$ Our findings, which are derived from self-report data, could represent a mixture of subclinical and clinical ED diagnoses and thus differ from cisgender samples because of overestimation of the true prevalence of clinical $\mathrm{AN}$ and $\mathrm{BN}$ in transgender adults. However, previous research has suggested that single item ED screens tend to underrather than overestimate the prevalence of EDs. ${ }^{12}$ Thus our results appear to support prior findings of increased ED prevalence in transgender adults relative to cisgender adults. However, the overall prevalence of ED in our study was lower than estimates in previous samples of transgender adults, possibly as a result of smaller sample size or differing sampling strategy. ${ }^{1,2}$

Study results should be interpreted within the limitations of these data. First, this secondary analysis of Project VOICE data was able to assess only selfreported and previously diagnosed $\mathrm{AN}$ or $\mathrm{BN}$, but not binge ED or other clinically significant DSM-5 disorders. As mentioned above, in population samples, while self-reported AN and BN single-item screens have very high specificity, they have lower sensitivity than longer questionnaires, ${ }^{12}$ meaning the true prevalence of EDs in this sample is likely underestimated. Second, Project VOICE collected data only on transgender adults, meaning that direct comparisons to ED prevalence in cisgender individuals were not possible. Third, due to the relatively small sample size and rare outcome, we were unable to control for several possible confounders, including socioeconomic status and gender-affirming medical care. Similarly, like any epidemiologic study, this analysis relies on untestable assumptions regarding the correctness of our model form and analytic strategy, and violations of these assumptions could result in bias in unpredictable directions. Finally, although Project VOICE included a diverse range of respondents from across Massachusetts, data were collected using convenience sampling, and over $80 \%$ of participants were identified through online access points, which may have resulted in selection bias and limited generalizability.
Despite limitations, this study highlights the nuanced relationship between gender identity and disordered eating and the need for further research on EDs in the transgender community. This study was, to the authors' knowledge, the first to evaluate differences in ED prevalence by gender identity subgroups among transgender participants. Large longitudinal studies with more comprehensive evaluations of EDs and potential mediating factors, particularly gender minority stress and socialization, are needed to elucidate the mental health trajectories of gender identity groups, including EDs. Future research should also evaluate how body image dissatisfaction relates to EDs in transgender people and whether this relationship is modified by assigned sex at birth. Given the dearth of data about EDs in transgender people, it is recommended that researchers include both assigned sex at birth and current gender identity questions in surveys to better understand ED risk by gender identity. ${ }^{28}$ The increased prevalence of reported EDs in this study of transgender adults indicates a need for more transgender-inclusive ED treatment and prevention services, as well as further research evaluating the efficacy of existing ED programming in transgender populations.

\section{Conclusions}

Gender nonconforming individuals assigned female at birth appear to have heightened lifetime risk of EDs relative to transgender individuals in other gender identity subgroups. Additional research into specific ED risk factors and gender-responsive intervention strategies are urgently needed. This study also highlights the variation in mental health needs across spectrums of gender identity, especially in transgender adults. Increasing the clinical providers' competence in gender identity represents an important public health approach to reducing health inequities beyond the gender binary.

\section{Author Disclosure Statement}

No competing financial interests exist.

\section{References}

1. Diemer EW, Grant JD, Munn-Chernoff MA, et al. Gender identity, sexual orientation, and eating-related pathology in a national sample of college students. J Adolesc Health 2015;57:144-149.

2. Ålgars M, Santtila P, Sandnabba NK. Conflicted gender identity, body dissatisfaction, and disordered eating in adult men and women. Sex Roles 2010;63:118-125.

3. Watson RJ, Veale JF, Saewyc EM. Disordered eating behaviors among transgender youth: probability profiles from risk and protective factors. Int J Eat Disord 2017;50:515-522. 
4. Guss CE, Williams DN, Reisner SL, et al. Disordered weight management behaviors and non-prescription steroid use in Massachusetts transgender youth. J Adolesc Health 2016;58:S102-S103.

5. Vocks S, Stahn C, Loenser K, Legenbauer T. Eating and body image disturbances in male-to-female and female-to-male transsexuals. Arch Sex Behav 2009;38:364-377.

6. Khoosal D, Langham C, Palmer B, et al. Features of eating disorder among male-to-female transsexuals. Sex Relation Ther 2009;24:217-229.

7. Bandini E, Fisher AD, Castellini G, et al. Gender identity disorder and eating disorders: similarities and differences in terms of body uneasiness. J Sex Med 2013:10:1012-1023.

8. Harrison J, Grant J, Herman JL. A gender not listed here: Genderqueers, gender rebels, and otherwise in the National Transgender Discrimination Survey. LGBTQ Public Policy Journal at the Harvard Kennedy School 2012;2:13-24.

9. Gordon AR, Austin SB, Krieger N, et al. "I have to constantly prove to myself, to people, that I fit the bill": Perspectives on weight and shape control behaviors among low-income, ethnically diverse young transgender women. Soc Sci Med 2016;165:141-149.

10. Miner $\mathrm{MH}$, Bockting WO, Romine RS, Raman S. Conducting Internet research with the transgender population: Reaching broad samples and collecting valid data. Soc Sci Comput Rev 2012;30:202-211.

11. Reisner SL, Hughto JM, Dunham EE, et al. Legal protections in public accommodations settings: a critical public health issue for transgender and gender-nonconforming people. Milbank Q 2015;93:484-515.

12. Keski-Rahkonen A, Sihvola E, Raevuori A, et al. Reliability of self-reported eating disorders: Optimizing population screening. Int J Eat Disord 2006; 39:754-762.

13. Reisner SL, Biello K, Rosenberger JG, et al. Using a two-step method to measure transgender identity in Latin America/the Caribbean, Portugal, and Spain. Arch Sex Behav 2014;43:1503-1514.

14. Centers for Disease Control and Prevention. 2012 Behavioral Risk Factor Surveillance System Questionnaire. 2012.

15. Allison PD. Missing data techniques for structural equation modeling. J Abnorm Psychol 2003;112:545.

16. Van Buuren S, Brand JPL, Groothuis-Oudshoorn CGM, Rubin DB. Fully conditional specification in multivariate imputation. J Stat Comput Simul 2006;76:1049-1064.

17. Lee KJ, Carlin JB. Multiple imputation for missing data: Fully conditional specification versus multivariate normal imputation. Am J Epidemiol 2010;171:kwp425.

18. Reisner SL, Conron K, Scout N, et al. Comparing in-person and online survey respondents in the US National Transgender Discrimination Survey: implications for transgender health research. LGBT Health 2014;1:98106.
19. Firth D. Bias reduction of maximum likelihood estimates. Biometrika 1993; 80:27-38.

20. Hughto JMW, Reisner SL, Pachankis JE. Transgender stigma and health: A critical review of stigma determinants, mechanisms, and interventions. Soc Sci Med 2015;147:222-231.

21. White Hughto JM, Pachankis JE, Willie TC, Reisner SL. Victimization and depressive symptomology in transgender adults: The mediating role of avoidant coping. J Couns Psychol 2017;64:41-51.

22. Grant JM, Mottet LA, Tanis J, et al. Injustice at Every Turn: A Report of the National Transgender Discrimination Survey. Washington: National Center for Transgender Equality, 2011.

23. Carter MJ. Gender socialization and identity theory. Soc Sci 2014;3:242263.

24. Ålgars $M$, Alanko K, Santtila P, Sandnabba NK. Disordered eating and gender identity disorder: A qualitative study. Eat Disord 2012;20:300-311.

25. Hepp U, Milos G. Gender identity disorder and eating disorders. Int J Eat Disord 2002;32:473-478.

26. Surgenor $L J$, Fear JL. Eating disorder in a transgendered patient: A case report. Int J Eat Disord 1998;24:449-452.

27. Hudson Jl, Hiripi E, Pope HG Jr, Kessler RC. The prevalence and correlates of eating disorders in the National Comorbidity Survey Replication. Biol Psychiatry 2007;61:348-358.

28. Reisner SL, Poteat T, Keatley J, et al. Global health burden and needs of transgender populations: a review. Lancet 2016;388:412-436.

Cite this article as: Diemer EW, White Hughto JM, Gordon AR, Guss C, Austin SB, Reisner SL (2018) Beyond the binary: differences in eating disorder prevalence by gender identity in a transgender sample, Transgender Health 3:1, 17-23, DOI: 10.1089/trgh.2017.0043.

\section{Abbreviations Used}

$\mathrm{AN}=$ anorexia nervosa

$\mathrm{BN}=$ bulimia nervosa

$E D=$ eating disorder

$\mathrm{FBGNC}=$ gender-nonconforming participants assigned female at birth FTM $=$ female-to-male

MBGNC $=$ gender-nonconforming participants assigned male at birth $\mathrm{MTF}=$ male-to-female

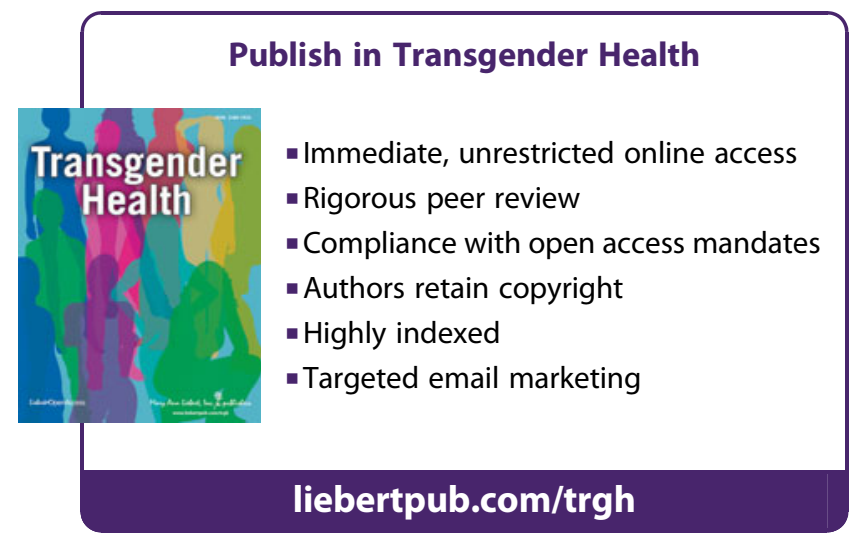

